\title{
Wegener's Granulomatosis with an Atypical Presentation as Acute Tonsillitis
}

\author{
Selçuk Arslan ${ }^{a} \quad$ Abdülcemal Ümit Işik ${ }^{a} \quad$ Sevdegül Mungan ${ }^{b} \quad$ Ahmet Ural $^{a}$ \\ Refik Ali Sarıc \\ Departments of a Otorhinolaryngology, ${ }^{b}$ Pathology, and ${ }^{\mathrm{C}}$ Immunology, School of Medicine, \\ Karadeniz Technical University, Trabzon, Turkey
}

\section{Key Words}

Tonsil · Wegener's granulomatosis · Tonsillar hypertrophy

\begin{abstract}
Wegener's granulomatosis (WG) is a rare necrotizing granulomatous vasculitis of unknown etiology commonly involving vessels, the upper and lower respiratory system and the kidneys. There are various symptoms and signs of WG due to a wide spectrum of involvement sites. The initial presentation of the disease as intraoral lesions is rare. In this paper, a case of WG that presented with a necrotic lesion confined to the right tonsil before other symptoms and signs occurred is described. The diagnosis was established based on the clinical presentation and the histopathological findings of the characteristic inflammatory pattern.
\end{abstract}

\section{Introduction}

Wegener's granulomatosis (WG) is a rare, chronic, relapsing, necrotizing vasculitis of small-to-medium-sized vessels, which usually involves the respiratory system and kidneys. Any organ other than this classical triad can be involved. The initial involvement of the upper and lower respiratory tract is known as limited WG, and if the disease is not treated, renal involvement often develops. This condition is known as generalized WG with poor prognosis 


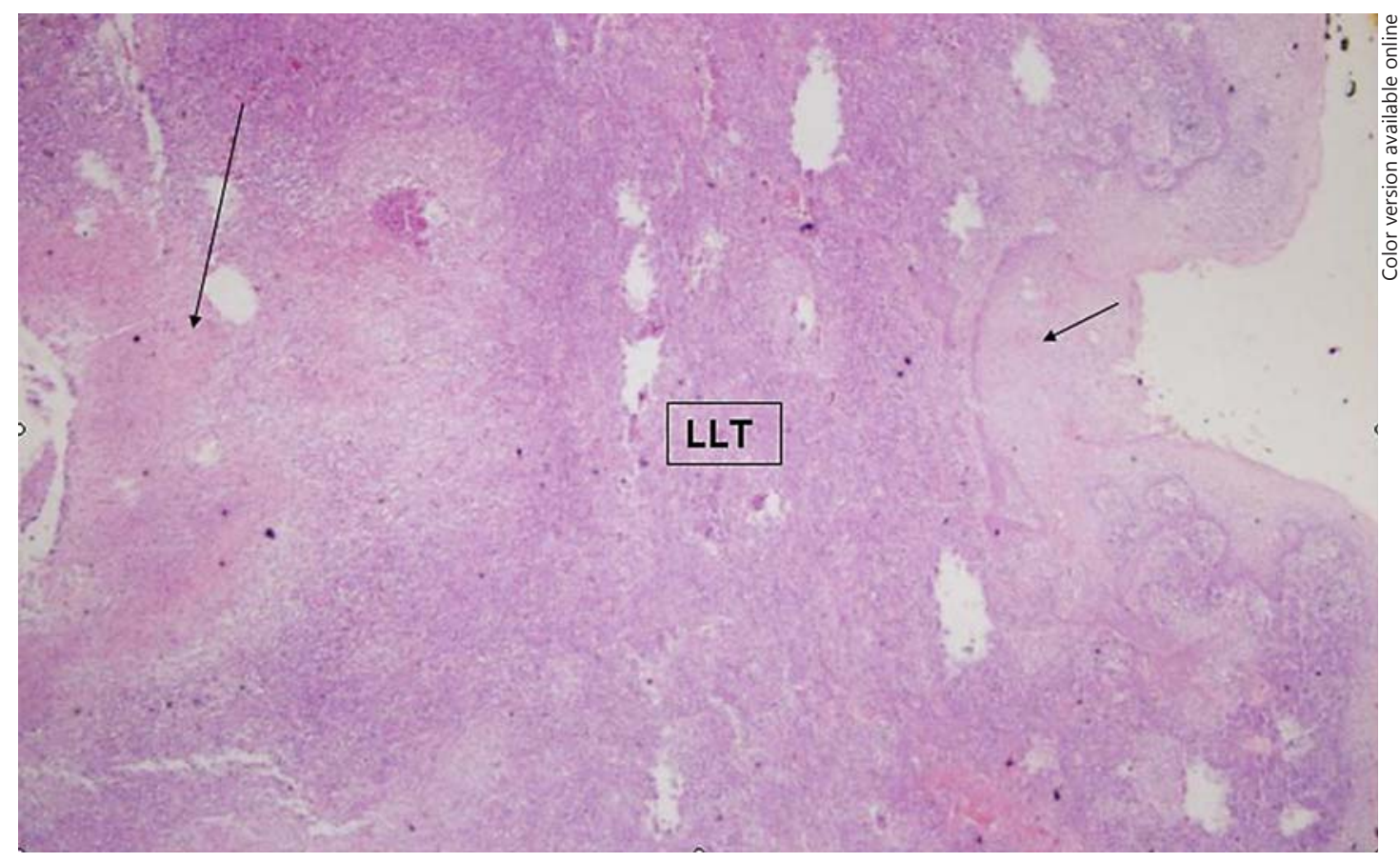

Fig. 1. The short arrow shows squamous epithelium of the tonsil, the long arrow the necrotic area and LLT stands for local lymphoid tissue. HE. $\times 40$.

due to renal failure secondary to glomerulonephritis [1]. The prognosis is much better for the limited form of WG, which has only respiratory involvement. Long-term remissions can be achieved with immunosuppressive therapy before irreversible organ failure occurs [2]. A superficial form of WG characterized by mucosal or cutaneous lesions and slow systemic involvement has also been described [3]. Oral lesions may be the initial manifestation of all three forms of WG, and systemic involvement can present within weeks or months after the development of oral lesions [4]. Although the oral manifestations in WG have been well described, the initial presentation with oral lesions is very rare, and presentation with oropharyngeal manifestation is even rarer. The reported case appeared to be in the form of early limited WG as the sole sign was tonsillar inflammation and no systemic symptoms were present on admission. In this paper, a rare case of WG with an atypical oropharyngeal involvement is described, and current diagnostic procedures and treatments are discussed.

\section{Case Report}

A 47-year-old male patient was admitted with a 9-day history of a right-sided sore throat, difficulty in swallowing and intermittent fever. No symptoms of cough, hemoptysis or nasal discharge were noted. The patient reported that the oral penicillin and analgesic treatment previously prescribed by the family physician had not relieved his symptoms. An oral examination revealed an inflamed right palatal tonsil with necrosis on the anterior surface. The left palatal tonsil, gingiva and the remainder of the oral cavity were normal in appearance. A slightly tender and mobile submandibular lymph node of $2 \times 1 \mathrm{~cm}$ on the right side was noticed upon palpation of the neck. An extraoral clinical examination showed no significant abnormalities otherwise. The patient was hospitalized with a preliminary diagnosis of acute tonsillitis, and intravenous ampicillin/sulbactam and anti-inflammatory treatment was initiated. The laboratory investigations revealed an increased erythrocyte sedimantation rate (ESR) at $98 \mathrm{~mm} / \mathrm{h}$ (normal range 0-20), C-reactive 
Fig. 2. Geographic necrosis surrounded by inflammatory cells. HE. $\times 200$.

Fig. 3. Large areas of fibrinoid necrosis in a deep pink color, stained with Mallory's trichrome $(\times 100)$.
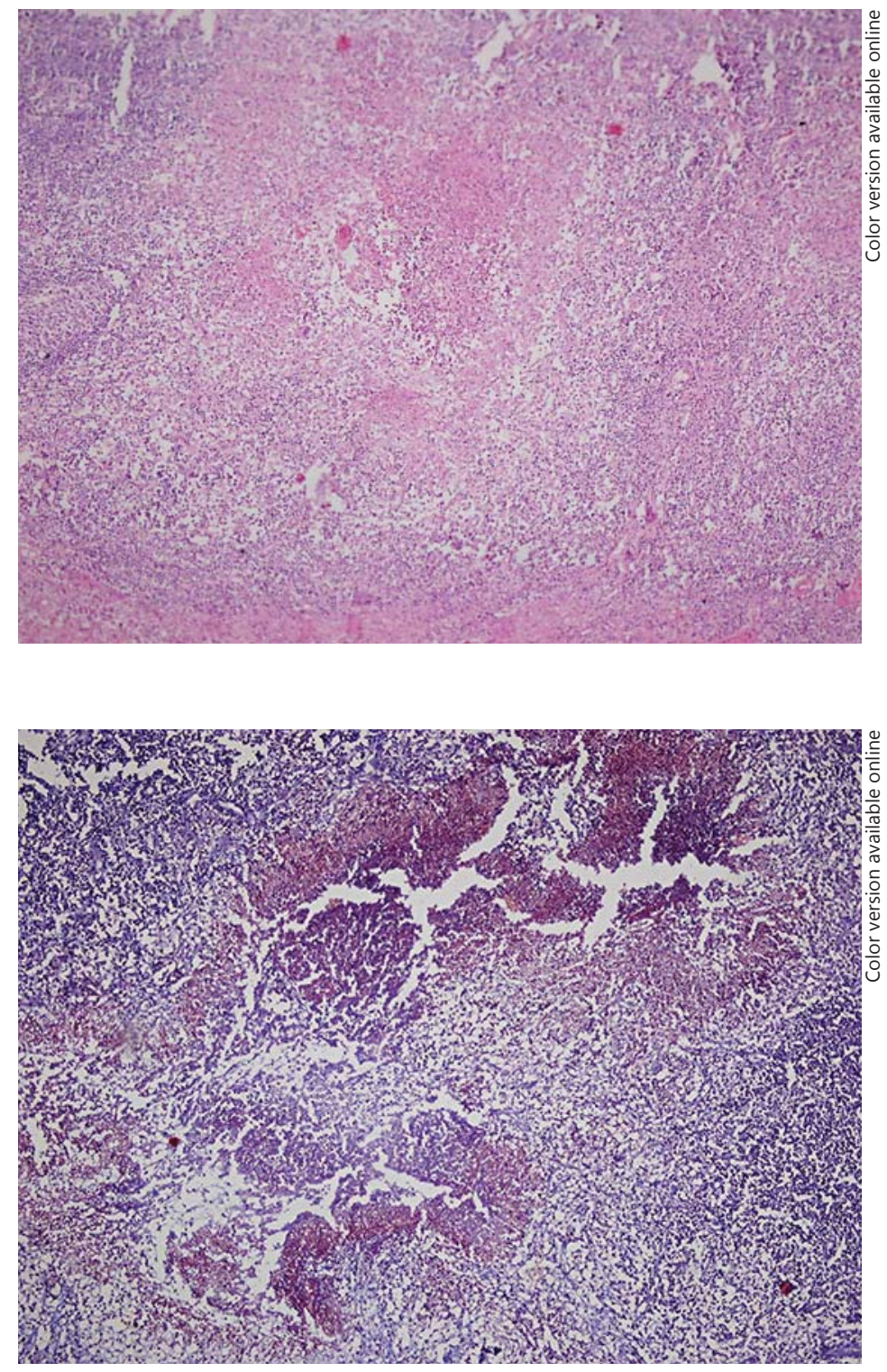

protein (CRP) at $15.7 \mathrm{mg} / \mathrm{l}$ (normal 0-0.5) and normal urea, liver function tests and electrolytes. Serology for cytoplasm-staining antineutrophil cytoplasmic antibody (c-ANCA) and perinuclear ANCA (p-ANCA) by immunofluorescence was negative, and viral serology (cytomegalovirus, Epstein-Barr virus, hepatitis B virus) proved negative. Chest radiography, abdomen ultrasonography and abdomen computerized tomography yielded normal findings. The patient did not respond to the 4-day treatment during hospital stay, and the inflammatory necrotic lesion in the tonsil turned to ulceration. With a provisional differential diagnosis of vasculitides, granulomatous disease and malignancy, histopathological examination of the lesion was performed. The right palatal tonsil was totally excised and submitted for histopathological examination.

Light microscopy demonstrated superficial squamous epithelium in a limited area because of ulceration (fig. 1). There were dense, active foci of chronic inflammation and geographical necrosis instead of local lymphoid tissue in the subepithelium (fig. 2). Some fibrinoid necrosis was shown histochemically by Mallory's trichrome staining (fig. 3). No hyphae, spore or bacterial growth was observed. There were no pathological findings related to malignancy. 
Arslan et al.: Wegener's Granulomatosis with an Atypical Presentation as Acute Tonsillitis

\section{Discussion}

WG is a systemic disease presenting as a triad of vascular, respiratory and renal involvement that can have a variable course from mild or indolent to a rapidly progressive disease leading to multiorgan failure when remained untreated [5].

WG most commonly presents with symptoms related to upper respiratory tract involvement. Sinusitis was reported to be the most common presenting symptom of WG (67\%) [6]. Up to one third of the patients complain of mucopurulant discharge, epistaxis, nasal obstruction, painful ulcers or septal perforation [7]. Coronal CT of paranasal sinuses is a useful diagnostic tool for the evaluation of a possible sinonasal disease. In the present case, paranasal CT revealed mucosal hypertrophy of maxillary sinuses, but the patient had no symptoms related to a possible sinonasal disease.

Renal disease occurs in approximately $18 \%$ of the patients at the time of presentation and indicates the generalized form of WG [8]. Renal biopsy can reveal a focal or segmental necrotizing glomerulonephritis, although it is not a specific finding for WG [9]. Urine analysis can detect microhematuria ( 5 red blood cells per high-power field) or red cell casts, which has almost a $100 \%$ positive predictive value for glomerulonephritis [7]. The urinary sediment was normal in our case.

Most patients with WG present with symptoms related to pulmonary involvement such as cough, hemoptysis and dyspnea. The involvement of the trachea or main bronchi can cause airway obstruction mimicking asthma [10]. The radiological examination may show various findings related to WG. Although multiple pulmonary nodules of $1-9 \mathrm{~cm}$ in size with welldefined borders and cavitation are characteristic for WG, a localized solitary mass mimicking a carcinoma or nonsegmental infiltrate that can rarely show progression can often be detected [11]. Pleural thickening and pleural effusion are the most frequent signs among pleural lesions that are present in $10 \%$ of the WG patients with pulmonary involvement [12]. Hilar lymphadenopathy or air bronchogram are rare pulmonary findings associated with WG.

Laboratory abnormalities in untreated patients include elevated ESR, white blood cell count and thrombocytes. Nearly $50 \%$ of the patients have normochromic normocytic anemia. Rheumatoid factor, antinuclear antibody and plasma immunoglobulin titers can vary. ANCA activity can be detected in all forms of vasculitides. A cytoplasmic staining pattern (c-ANCA) has been predominantly associated with active WG in contrast to p-ANCA, which has been frequently associated with microscopic polyangiitis, other vasculitides, idiopathic necrotizing and crescentic glomerulonephritis and other diseases [13]. In the presence of c-ANCA, specificity has been reported to be up to $97 \%$ and sensitivity for $W G$ is $90 \%$, and it is a rather important aid in diagnosing limited or atypical forms of WG [14]. However, when the disease is not severe, sensitivity may be as low as $66 \%$ [13]. The positivity of c-ANCA decreases as the disease enters remission. Therefore, monitoring c-ANCA levels offers a helpful tool to differentiate disease relapses from intercurrent infections [2]. In the present case, the remarkable laboratory results included raised ESR levels of $98 \mathrm{~mm} / \mathrm{h}$ and C-reactive protein values of $15.7 \mathrm{mg} / \mathrm{l}$. The c-ANCA and p-ANCA values were negative in our case. This might be due to mild disease activity because the patient presented with the early, limited form of WG.

Histologically, there is mixed inflammation centered on blood vessels. The lesions are granulomatous with acute and chronic inflammatory cells. Areas of necrosis and multinucleated giant cells can be observed. The similar histopathological findings were detected in our case.

Oral lesions rarely present in WG. Duna et al. [7] reported that oral cavity lesions were the presenting sign for only $2 \%$ of the patients they examined. Several oral manifestations of WG have been described, mostly including a characteristic hyperplastic granular gingivitis called 'strawberry gums' and less commonly palatal ulceration or perforation and lip ulcers 
Arslan et al.: Wegener's Granulomatosis with an Atypical Presentation as Acute Tonsillitis

[4]. The presentation of WG with inflammation or ulceration is limited to tonsillar tissue. To the best of our knowledge, isolated involvement of tonsils by WG has not been previously reported. Singh et al. [15] reported a similar case presenting with a necrotic mass in the right tonsillar area, with extensive ulceration of the right side of the soft palate and vasculitic lesions on the hands and feet. Our case showed a unique presentation of tonsillar inflammation without any accompanying symptom.

Untreated WG has a poor prognosis with an $82 \%$ mortality rate in the first year [7]. Steroids and cyclophosphamide (CYC) are the mainstays of treatment, and most patients respond immediately within 1 week. The treatment is targeted to induce remission with oral prednisone $1 \mathrm{mg} / \mathrm{kg} /$ day and CYC $2-3 \mathrm{mg} / \mathrm{kg} /$ day [8]. The treatment begins with prednisone $1 \mathrm{mg} / \mathrm{kg} /$ day and is continued until remission is achieved (usually after 1 month). Afterwards, it is tapered gradually to an alternate-day dose at 3 months and then discontinued. Along with the tapering of the steroid, CYC $2 \mathrm{mg} / \mathrm{kg} /$ day is introduced and often continues for up to 1 year after the patient achieved remission [7]. Our patient received a standard treatment consisting of prednisone and CYC. The treatment protocol consisted of oral prednisone $1 \mathrm{mg} / \mathrm{kg} /$ day for $1 \mathrm{month}$ and CYC $2 \mathrm{mg} / \mathrm{kg} /$ day for 1 year. Prednisone was tapered after 1 month and discontinued after 6 weeks. The patient was free of symptoms 1 year later.

WG can present with atypical manifestations, as reported in the present case. WG should be considered in the differential diagnosis of any intraoral ulcer of unknown etiology to start proper treatment as soon as possible to improve the outcome of this potentially lethal disease.

\section{Disclosure Statement}

The authors have no conflicts of interest to disclose.

\section{References}

1 Singha V, Dhingraa R, Sharma RK, Narwal A, Gupta A: Wegener's granulomatosis presenting as unhealing palatal ulceration - a case report. J Oral Maxillofac Surg 2012;24:218-221.

- 2 Rahilly G, Rahilly M: A case of palatal Wegener's granulomatosis. Oral Dis 2000;6:259-261.

3 Neville BW, Damm DD, Allen CM, Bouquot JE: Oral and Maxillofacial Pathology, ed 3. Philadelphia, Saunders, 2010, pp 188-193, 287, 471-472, 602.

4 Handlers JP, Waterman J, Abrams AM, Melrose RJ: Oral features of Wegener's granulomatosis. Arch Otolaryngol 1985;111:267-270.

- 5 Lilly J, Juhlin T, Lew D, Vincent S, Lilly G: Wegener's granulomatosis presenting as oral lesions: a case report. Oral Surg Oral Med Oral Pathol Oral Radiol Endod 1998;85:153-157.

- 6 Hoffman GS, Kerr GS, Leavitt RY: Wegener's granulomatosis: an analysis of 158 patients. Ann Intern Med 1992; 116:488-498.

- 7 Duna GF, Galperin C, Hoffman GS: Wegener's granutomatosis. Rheum Dis Clin North Am 1995;21:949-986.

- 8 Fauci AS, Haynes BF, Katz R: Wegener's granulomatosis: prospective clinical and therapeutic experience with 85 patients for 21 years. Ann Intern Med 1983;98:76-85.

- 9 Aasarod K, Bosted L, Hammerstrom J, Jorstad S, Iverson BM: Renal histopathology and clinical course in 94 patients with Wegener's granulomatosis. Nephrol Dial Transplant 2001;16:953-960.

-10 Daum DE, Specks AM, Colby TV: Tracheobronchial involvement in Wegener's granulomatosis. Am J Respir Crit Care Med 1995;151:522-526.

11 Chiang IC, Liu GC, Sheu RS, Kuo YT: High resolution CT of Wegener's granulomatosis - case reports. Kaohsiung J Med Sci 2001;17:221-225.

$\checkmark 12$ Diot E, Lavigne C, Renjard L, Asquier E, Valentin JF, Legras A, Guilmot JL, Lemarié E, Diot P: Wegener's disease mimicking acute infectious pleurisy (in French). Rev Pneumol Clin 2000;56:265-268.

13 Rao JK, Weinberger M, Oddone EZ, Allen NB, Landsman P, Feussner JR: The role of antineutrophil cytoplasmic antibody (c-ANCA) testing in the diagnosis of Wegener's granulomatosis. Ann Intern Med 1995;123:925-932.

14 Vacchi SM, Frasca G: Clinical significance of 'ANCA' in the diagnosis of Wegener's granulomatosis: 8 years of experience. Acta Otorhinolaryngol Ital 1998;18:239-248.

15 Singh S, Balakrishnan C, Bajan K, Bhaduri A: Wegener's granulomatosis presenting as a tonsillar mass. J Assoc Physicians India 2009;57:603. 